\title{
High-resolution measurement of cloud microphysics and turbulence at a mountaintop station
}

\author{
H. Siebert ${ }^{1}$, R. A. Shaw ${ }^{2}$, J. Ditas ${ }^{5}$, T. Schmeissner ${ }^{1}$, S. P. Malinowski ${ }^{3}$, E. Bodenschatz ${ }^{4}$, and H. Xu ${ }^{4}$ \\ ${ }^{1}$ Leibniz Institute for Tropospheric Research, Leipzig, Germany \\ ${ }^{2}$ Department of Physics, Michigan Technological University, Michigan, USA \\ ${ }^{3}$ Institute of Geophysics, Faculty of Physics, University of Warsaw, Warsaw, Poland \\ ${ }^{4}$ Max Planck Institute for Dynamics and Self-Organization (MPIDS), Göttingen, Germany \\ ${ }^{5}$ Max Planck Institute for Chemistry, Mainz, Germany \\ Correspondence to: H. Siebert (siebert@tropos.de)
}

Received: 16 December 2014 - Published in Atmos. Meas. Tech. Discuss.: 15 January 2015

Revised: 18 June 2015 - Accepted: 23 June 2015 - Published: 13 August 2015

\begin{abstract}
Mountain research stations are advantageous not only for long-term sampling of cloud properties but also for measurements that are prohibitively difficult to perform on airborne platforms due to the large true air speed or adverse factors such as weight and complexity of the equipment necessary. Some cloud-turbulence measurements, especially Lagrangian in nature, fall into this category. We report results from simultaneous, high-resolution and collocated measurements of cloud microphysical and turbulence properties during several warm cloud events at the Umweltforschungsstation Schneefernerhaus (UFS) on Zugspitze in the German Alps. The data gathered were found to be representative of observations made with similar instrumentation in free clouds. The observed turbulence shared all features known for high-Reynolds-number flows: it exhibited approximately Gaussian fluctuations for all three velocity components, a clearly defined inertial subrange following Kolmogorov scaling (power spectrum, and second- and thirdorder Eulerian structure functions), and highly intermittent velocity gradients, as well as approximately lognormal kinetic energy dissipation rates. The clouds were observed to have liquid water contents on the order of $1 \mathrm{~g} \mathrm{~m}^{-3}$ and size distributions typical of continental clouds, sometimes exhibiting long positive tails indicative of large drop production through turbulent mixing or coalescence growth. Dimensionless parameters relevant to cloud-turbulence interactions, the Stokes number and settling parameter are in the range typically observed in atmospheric clouds. Observed fluctuations in droplet number concentration and diameter suggest a pref-
\end{abstract}

erence for inhomogeneous mixing. Finally, enhanced variance in liquid water content fluctuations is observed at high frequencies, and the scale break occurs at a value consistent with the independently estimated phase relaxation time from microphysical measurements.

\section{Introduction}

Measurements of detailed interactions between turbulence and cloud processes are challenging. Airborne measurements allow for the most flexibility in going to the clouds of interest, but sample times are limited and measurements are inherently Eulerian in nature. We have investigated the suitability of making simultaneous and collocated cloud and turbulence measurements from a mountaintop research station, with the aim of characterizing the fine-scale turbulence and cloud microphysical properties. From a ground-based station it is possible to measure for extended periods of time, and it also becomes feasible to perform measurements involving Lagrangian tracking of small volumes of cloudy air. The main question in this context is in which ways the sampled clouds are influenced by the presence of the laboratory and the mountain, and whether the observed small-scale features are still representative of free-atmospheric clouds.

The context of this study specifically addresses cloudturbulence interactions. In a companion paper (Risius et al., 2015) the seasonal cloud and flow conditions are discussed. In this paper we consider the observed turbulence structure 
at scales well in the inertial subrange down to about 10 times the Kolmogorov scale (typically $1 \mathrm{~mm}$ or so) and cloud microphysical properties and compare them to conditions observed in free clouds with similar instrumentation. Specifically, we present high-spatial-resolution measurements of liquid water content, droplet diameter, and turbulent velocity and temperature fluctuations. The observations were performed at the Umweltforschungsstation (environmental research station) Schneefernerhaus (UFS) in summer and fall 2009, and again in summer 2011. The measurements are analyzed and interpreted in a manner similar to data recorded in cumulus and stratocumulus clouds by the ACTOS (Airborne Cloud Turbulence Observation System) platform (Siebert et al., 2006a) in order to compare the two approaches.

The UFS is located in the German Alps near the top of Zugspitze $\left(47^{\circ} 25^{\prime} 00^{\prime \prime} \mathrm{N}, 10^{\circ} 58^{\prime} 46^{\prime \prime} \mathrm{E}\right)$, the highest mountain in Germany (2962 ma.s.1.). The station is situated on the north side of the glacier and near the top of Zugspitze, at a height of about $2650 \mathrm{~m}$. The UFS is a nine-story building, constructed into the southern flank of the Zugspitze, and it experiences frequent immersion in clouds (cf. upper panel of Fig. 1). Due to the local topography, the winds measured at the UFS are primarily in the east-west direction. The near uniformity of wind direction is a significant advantage for measurements because it allows instruments to be pointed in one fixed direction (e.g., see Fig. 1). Further details on the turbulence and flow characteristics under cloudy conditions at the UFS are given by Risius et al. (2015).

\section{Experimental setup}

Measurements of fine-scale turbulence and cloud microphysical properties in a Eulerian reference frame were performed from a fixed $3 \mathrm{~m}$ high mast with various measurement instruments (cf. Fig. 1). The mast was situated on the ninth-floor measurement platform during the first campaign (3-22 August 2009). The distance to the edge of this floor was about $2 \mathrm{~m}$. The ultrasonic anemometers (hereafter called "sonics") at the mast were orientated westward during both measurement periods.

The exact setup of the mast with the heights of the individual sensors is shown in Fig. 1. Two sonics of Solent HS type manufactured by Gill Ltd, Lymington, UK, were mounted at heights of 1.80 and $2.55 \mathrm{~m}$ above the terrace. The measurement of the three-dimensional wind velocity vector and the virtual temperature are based on transit time measurements of ultrasonic pulses traveling between two transducers (for one velocity vector component) with and against the wind. The temporal resolution of the sonics is $100 \mathrm{~Hz}$, and the measuring resolution of the wind velocity and temperature are $\Delta u=0.01 \mathrm{~m} \mathrm{~s}^{-1}$ and $\Delta T=0.01 \mathrm{~K}$, respectively (see Siebert and Muschinski (2001) for more details on the Solent HS). An ultra-fast thermometer (UFT) and a onecomponent hot-wire anemometer were fixed at a height of

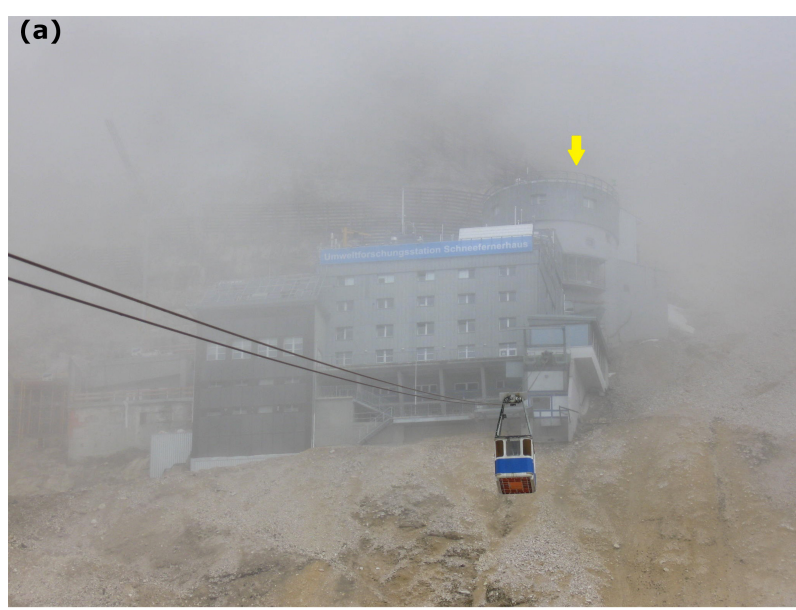

(b)

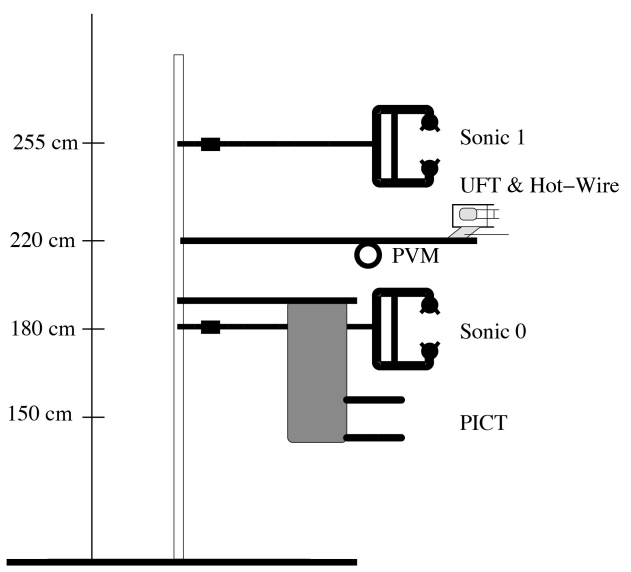

Figure 1. (a) The picture shows the UFS partly immersed in clouds. The location of the experimental setup on the ninth floor is indicated by the yellow arrow. (b) A schematic of the mast equipped with two sonics, an ultra-fast thermometer (UFT), a one-component hotwire anemometer, a particle volume monitor (PVM) for liquid water content measurements, and a phase Doppler interferometer (PICT) is shown in the lower panel, including the individual measurement heights above the terrace.

$2.20 \mathrm{~m}$. The UFT is based on a $2.5 \mu \mathrm{m}$ resistance wire protected against droplet impaction (cf. Haman et al., 1997), and the hot-wire anemometer uses a constant-temperature approach (Comte-Bellot, 1976). Both instruments are sampled at a rate of $1 \mathrm{kHz}$.

Cloud microphysical variables were also measured from the mast. A particle volume monitor (PVM-100A; see Gerber, 1991) was positioned beside the UFT to measure the liquid water content (LWC) and the particle surface area (PSA). The intensity of laser light diffracted by a cloud droplet ensemble in a given measurement volume is related to the absolute volume concentration through the use of a custom transmission filter in front of the detector. Liquid water contents are measurable within the full range of those observed in typical clouds (artifacts are known to be present for low concentrations of large droplets such as those encountered in pris- 
tine environments, but this is not a problem for the microphysical conditions encountered at the UFS). Cloud droplet size distributions were measured with a phase Doppler interferometer (PICT), located at a height of $1.50 \mathrm{~m}$ above ground. The measurement principle is based on heterodyne detection of Doppler-shifted light from individual droplets, resulting in a robust measurement of the droplet diameter and a single component of the droplet velocity vector (Chuang et al., 2008).

\section{Data and analysis}

The first part of this section presents a characterization of the turbulent flow under cloud-free conditions. The data were taken on 18 August 2009 starting at 22:36 LT (local time). After characterizing the mean flow, we consider how closely the turbulence follows the classical picture of homogeneous, isotropic turbulence at the fine scale by looking at velocity fluctuations, scaling for velocity power spectra and Eulerain structure functions, and velocity gradient and energy dissipation rate distributions. Here, the absence of cloud droplets allows hot-wire data to be used with full resolution $(\approx 4 \mathrm{~mm})$ because no de-spiking algorithm has to be applied to remove spikes in the data due to droplet impaction (Siebert et al., 2007). From this high-resolution data we address the question of whether on the smallest observable scales the turbulence is similar to that observed in the free atmosphere, e.g., under conditions without the direct influence of the ground.

The second part of this section presents microphysical data from two episodes when the UFS was immersed in clouds. The microphysical properties of the clouds and, simultaneously, the turbulent properties of the flow are characterized, and a comparison with conditions encountered in clouds occurring in the free atmosphere is made. Ultimately, this depends not only on the direct quantities measured but also on the dimensionless parameter space of relevance to cloud-turbulence interactions (Siebert et al., 2010a); so both are presented here. For this analysis, we have selected two episodes with quite different microphysical conditions in terms of the mean droplet diameter but a rather similar mean degree of turbulence. Both the turbulence and microphysical properties are compared to recent cloud measurements made with similar instrumentation.

\subsection{Mean flow characteristics}

Figure 2 shows a $1 \mathrm{~h}$ long time series of the horizontal wind velocity $U=\sqrt{u^{2}+v^{2}}$ and the wind direction $d$; here $u$ and $v$ are the two horizontal wind velocity vector components in the sonic reference frame. During the measurements the prevailing wind was from westerly directions $\left(300^{\circ}\right)$; that is, the mean flow was along the mountain contour. Periods with $d \leq 200$ and $\geq 330^{\circ}$ are probably influenced by up- and downhill flow and should be interpreted with caution.

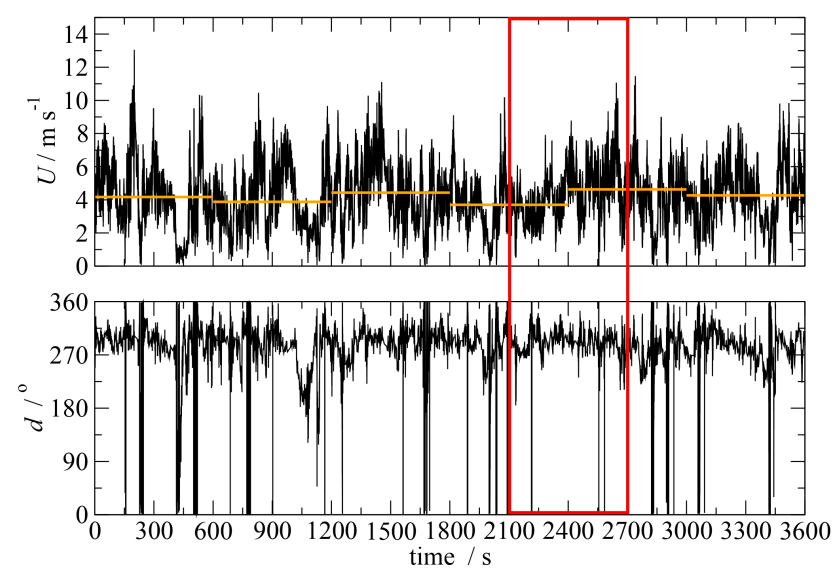

Figure 2. Time series of horizontal wind velocity $U=\sqrt{u^{2}+v^{2}}$ and wind direction $d$. The solid orange lines mark the mean value for each of the six $10 \mathrm{~min}$ long subrecords. Data were measured with the upper sonic (S1) about $2.55 \mathrm{~m}$ above the surface.

The measurements were conducted under cloud free conditions, but typical also for the two cloud cases analyzed in Sect. 3.3. The time period was selected to exhibit typical conditions when the flow properties were nearly statistically stationary in terms of the wind direction, although the fluctuations were increasing slightly with time. During this period, $U$ ranged from nearly 0 to $13 \mathrm{~m} \mathrm{~s}^{-1}$ with a mean value of $\bar{U}=4.2 \mathrm{~m} \mathrm{~s}^{-1}$ and a standard deviation of $\sigma_{U}=1.9 \mathrm{~m} \mathrm{~s}^{-1}$. However, it should be noted that the mean turbulence intensity $\sigma_{U} / \bar{U} \approx 0.45$ indicates that the validity of Taylor's hypothesis for the entire record is questionable. Therefore, for further analysis the record will be divided into smaller nonoverlapping $10 \mathrm{~min}$ long subrecords with turbulence intensity well below 0.5 to fulfill the conditions for the validity of Taylor's hypothesis (e.g., Willis and Deardorff, 1976). The mean value for each of the six subrecords is included in Fig. 2 as a solid orange line.

The velocity fluctuations $u^{\prime}(t)=u(t)-\bar{u}$ were determined from $10 \mathrm{~min}$ long subrecords, where $\bar{u}$ is the mean value of the subrecord. For each subrecord, a matrix transformation was applied such that $\bar{v}=\bar{w}=0$ and $\bar{u}$ is along the mean flow. In Fig. 3 the probability density functions (PDFs) of the normalized velocity components $u_{i}^{\prime} / \sigma_{u_{i}}$ are presented. Each PDF includes the data of all six subrecords. For reference, a Gaussian distribution is shown. The PDFs of all three velocity components can be well approximated by a Gaussian distribution, in agreement with observations for homogeneous and locally isotropic turbulence (Davidson, 2004).

\subsection{Hot-wire measurements: fine-scale turbulence}

The fine-scale turbulence properties are illustrated using the hot-wire data indicated by the red box in Fig. 2. The onedimensional data were sampled at $f_{s}=1 \mathrm{kHz}$, resulting in a mean spatial resolution of $\approx 4 \mathrm{~mm}$. The mean wind velocity 


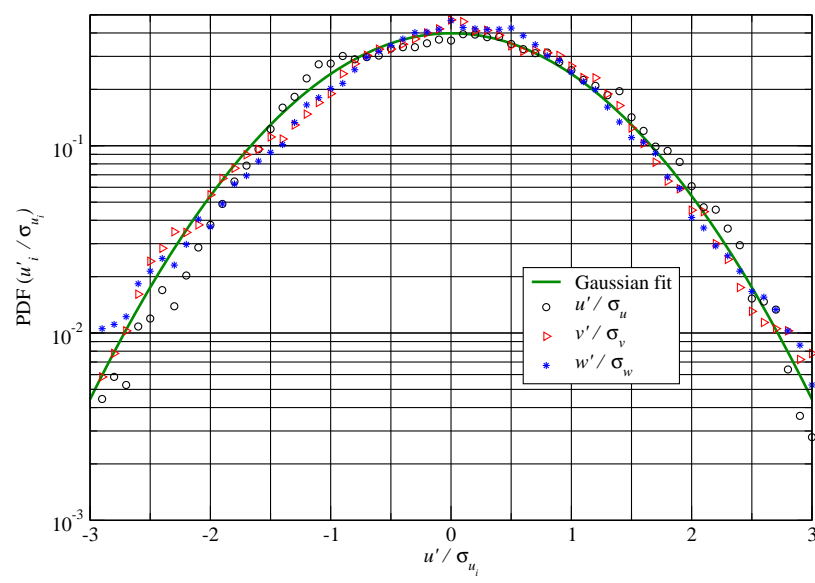

Figure 3. Probability density functions of normalized velocity component fluctuations $u_{i}^{\prime} / \sigma_{u_{i}}$ for the upper sonic. The fluctuations $u^{\prime}$ are calculated for each of the six subrecords by subtracting the mean value of the subrecord. The PDF includes the data from all six subrecords. A Gaussian distribution is given as a reference.

of this subrecord is $\langle u\rangle=4.2 \mathrm{~m} \mathrm{~s}^{-1}$ with a standard deviation of $\sigma_{u}=1.4 \mathrm{~m} \mathrm{~s}^{-1}$, which yields a turbulence intensity of $\sigma_{u} /\langle u\rangle \approx 0.33$, slightly below the critical value of 0.5 for the validity of Taylor's hypothesis (Willis and Deardorff, 1976).

In Fig. 4 the one-dimensional spectrum $f \cdot S_{u}(f)$ is plotted. The spectrum shows a clear inertial subrange scaling in the frequency range of 1 to $60 \mathrm{~Hz}$ (approximately $4 \mathrm{~m}$ to $7 \mathrm{~cm}$ ). Figure 8 of Risius et al. (2015) shows that the inertial subrange actually extends to approximately $f=10^{-2} \mathrm{~Hz}$. A linear fit was applied for that region (solid black line), yielding a slope of -0.64 , which is close to the theoretical value of $-2 / 3$. For frequencies below $1 \mathrm{~Hz}$, the spectrum scatters due to statistical noise, and for $f>60 \mathrm{~Hz}$ the spectrum drops off due to the increasing influence of dissipation. This effect will be discussed later after the structure functions have been introduced.

The turbulent energy dissipation rate per unit mass $\varepsilon$ can be estimated from the inertial range portion of the spectrum using

$S(f)=\alpha\left(\frac{\bar{U}}{2 \pi}\right)^{2 / 3} \varepsilon^{2 / 3} f^{-5 / 3}$,

where $\alpha$ is a universal constant $(\alpha \approx 0.5)$, and the factor $\bar{U} / 2 \pi$ is due to the conversion of the spectrum from wave number to frequency space. This approach leads to a mean of $\varepsilon=8.5 \times 10^{-2} \mathrm{~m}^{2} \mathrm{~s}^{-3}$, yielding a Kolmogorov length scale of $\eta=\left(v^{3} / \varepsilon\right)^{1 / 4} \approx 0.4 \mathrm{~mm}$. This is still 1 order of magnitude below the resolution of our measurements but typical for highly turbulent parts of atmospheric clouds.

As an alternative method for estimating the mean energy dissipation rate, we consider the following relationships for $n$th order structure functions of the longitudinal component

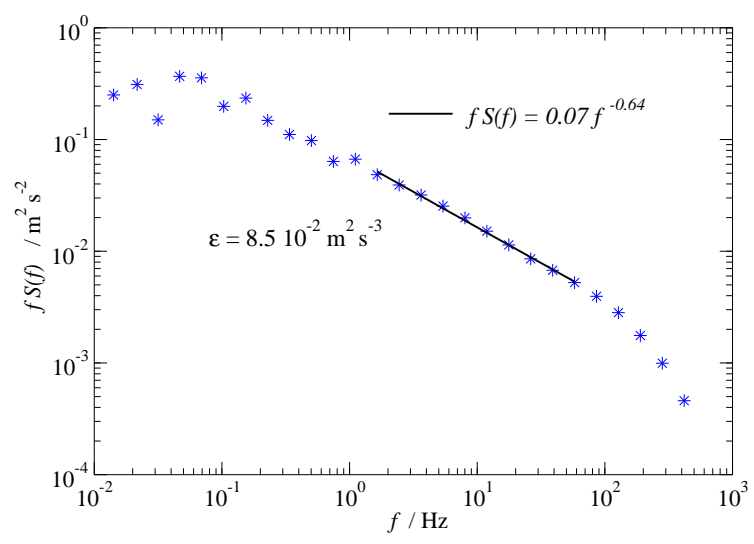

Figure 4. Power spectral density function $S(f)$ multiplied with frequency $f$ as derived from a 10 min subrecord (as indicated with in red in Fig 2) of a one-component hot-wire. The sampling frequency is $1 \mathrm{kHz}$. From an inertial subrange fit (see black solid line), a mean energy dissipation rate of $\varepsilon \approx 8.5 \times 10^{-2} \mathrm{~m}^{2} \mathrm{~s}^{-3}$ was estimated.

of the velocity vector $D_{(n)}=<(u(x+r)-u(x))^{n}>$ :

$D_{(2)}(r)=C \varepsilon^{2 / 3} r^{2 / 3}$

where $C$ has been found empirically to be approximately 2 , and

$D_{(3)}(r)=-\frac{4}{5} \varepsilon r$.

Both functions are plotted in Fig. 5 together with their compensated form. The horizontal line marks the mean $\bar{\varepsilon} \approx 8.5 \times$ $10^{-2} \mathrm{~m}^{2} \mathrm{~s}^{-3}$ as derived from the spectrum, which agrees within about $10 \%$ compared to the two estimates from the structure function relationships.

The wave number $k$ at which dissipation effects are manifested in the power spectrum has been shown to be approximately $k=(2 \pi f) / \bar{U}=1 /(8 \eta)$ (Monin and Yaglom, 2007). With a mean flow velocity of $U=4 \mathrm{~m} \mathrm{~s}^{-1}$ and $\eta=0.4 \mathrm{~mm}$ we find $f \approx 50 \mathrm{~Hz}$, which agrees well with the observed power spectrum in Fig. 4. For the second-order structure function the same authors suggest that for $r \leq 50 \eta$ dissipation effects become significant, which agrees with our observation in Fig. 5.

Small-scale turbulence at high Reynolds numbers is characterized by its intermittent nature, that is, periods of comparably small differences alternating with bursts of significantly increased gradients. This behavior can be better seen in the local velocity gradients $\mathrm{d} u / \mathrm{d} x$. Here, $\mathrm{d} u=u_{i+1}-u_{i}$ and $\mathrm{d} x=\mathrm{d} t \cdot 1 / 2\left(u_{i}+u_{i+1}\right)$, where $\mathrm{d} t=10^{-3} \mathrm{~s}$ is the time resolution of the measurements. The normalized gradients $\gamma=$ $((\mathrm{d} u / \mathrm{d} x)-\overline{\mathrm{d} u} / \mathrm{d} x) / \sigma_{\mathrm{d} u} / \mathrm{d} x$ are shown in the upper panel of Fig. 6. Values up to 15 times the standard deviations are observed quite frequently, which is a typical feature of intermittency. During the time period between $t=2350$ and $2550 \mathrm{~s}$, bursts seem to occur quasi-periodically, with a pe- 

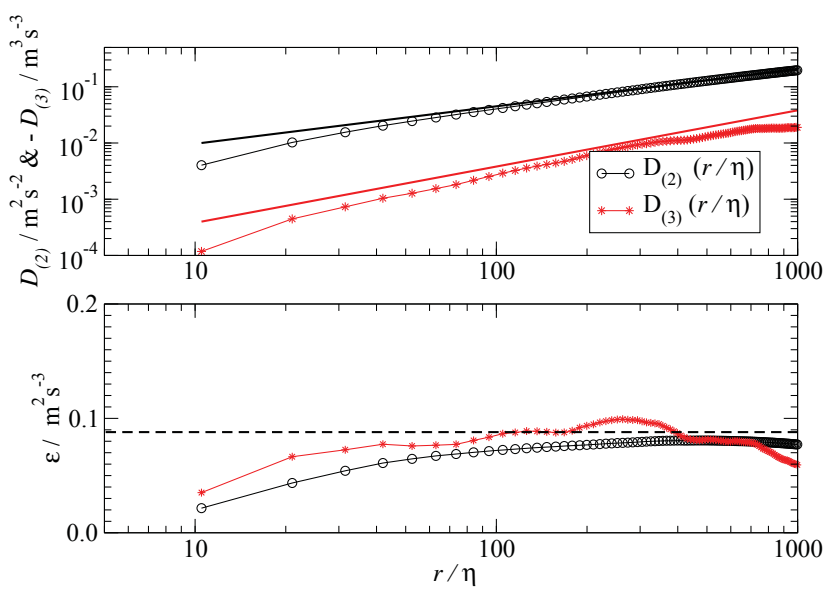

Figure 5. Second- and third-order structure functions derived from hot-wire measurements with $1 \mathrm{~ms}$ time resolution (upper panel) and compensated structure functions as a function of $r / \eta$ yielding the energy dissipation rate $\varepsilon$ (bottom panel). The solid lines represents $r^{2 / 3}$ and $r$ for reference. The structure functions are based on the same data set as in Fig. 4. The dashed horizontal line in the lower panel marks the energy dissipation rate derived from the power spectrum.

riod of about $50 \mathrm{~s}$. If so, this might be a signature of locally generated eddies (Whitemann, 2000). A longer time series would be required in order to unambiguously identify an actual periodicity. The corresponding PDF in a semilogarithmic plot (lower panel of Fig. 6) shows significantly enhanced tails compared to a Gaussian distribution, with the tails approximately exhibiting an exponential shape. Skewness $S=\overline{\gamma^{3}}=-0.4$ and kurtosis $K=\overline{\gamma^{4}}=20$ were calculated. Wind-tunnel observations (Gylfason et al., 2004) suggest a power-law dependency of $S$ and $K$ on the Taylor microscale Reynolds number $R e_{\lambda}=\sigma_{u}^{2} \sqrt{15 /(\varepsilon v)}$. Using their formulae for the observed $R_{\lambda}=6200$, we obtain $S=$ $-0.33 R e_{\lambda}{ }^{0.09} \approx-0.7$ and $K=0.91 R e_{\lambda}{ }^{0.39} \approx 28$, which are in qualitative agreement with the directly calculated $S$ and $K$. It has to be considered that the hot-wire measurements have a spatial resolution of about $10 \cdot \eta$ and the smallest relevant scales cannot be resolved, which can explain the possible underestimation of $S$ and $K$. In free-atmospheric clouds $K \approx 8$ has been observed at scales $\sim 20 \cdot \eta$ with strongly increasing values with increasing resolution (Siebert et al., 2010b).

Finally, we present the PDF of $\varepsilon_{\tau}$ derived from secondorder structure functions (cf. Eq. 2). Here, each $\varepsilon_{\tau}$ is estimated from $100 \mathrm{~ms}$ long subrecords; that is, the time series of $\varepsilon_{\tau}$ has a frequency of $10 \mathrm{~Hz}$. Figure 7 shows the PDF of $\ln \left(\varepsilon_{\tau}\right)$ together with a Gaussian fit in a semi-logarithmic plot. The good agreement of the measurements and the Gaussian distribution indicate that $\varepsilon_{\tau}$ is approximately lognormal, in accordance with the refined similarity theory of Kolmogorov (1962). Similar results have been found also for turbulent clouds (Siebert et al., 2010a, b), which supports the conclu-
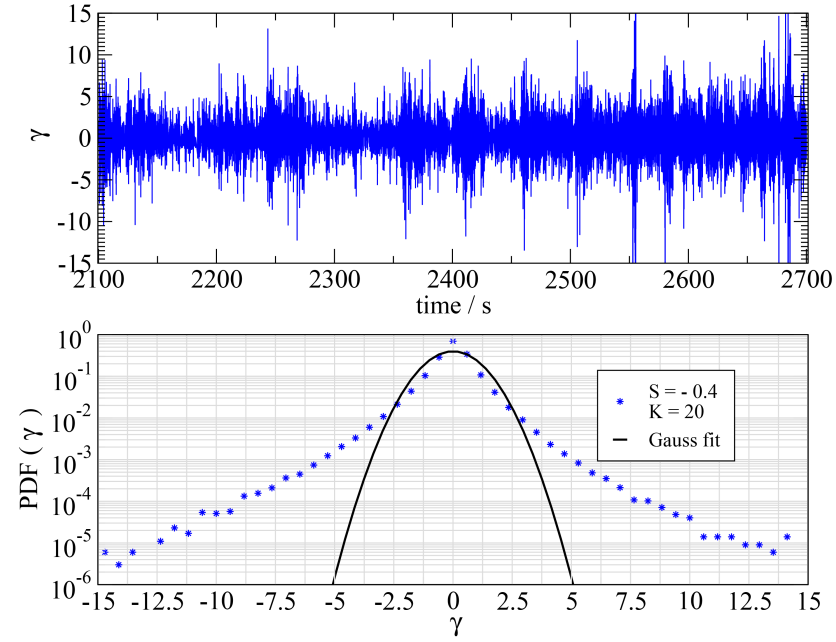

Figure 6. Upper panel: $10 \mathrm{~min}$ subrecord of normalized velocity gradients $\gamma=((\mathrm{d} u / \mathrm{d} x)-\overline{\mathrm{d} u / \mathrm{d} x}) / \sigma_{\mathrm{d} u / \mathrm{d} x}$ obtained from the hotwire data (same data as in Fig. 4). Lower panel: the corresponding PDF in semi-logarithmic plot with a Gaussian distribution for reference. The skewness $S=-0.4$ and the kurtosis $K=20$.

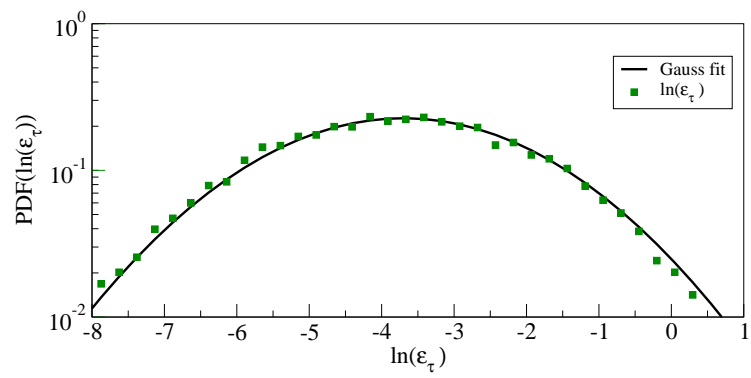

Figure 7. Probability density function (PDF) of $\ln \left(\varepsilon_{\tau}\right)$ with $\tau=$ $0.1 \mathrm{~s}$ estimated from the same data as in Fig. 4. A Gaussian distribution is plotted as a reference.

sion that on small scales the turbulence at the UFS is representative of turbulence observed in "free" clouds, in spite of the possibility that on larger scales the turbulence differs in terms of isotropy and shear due to the influence of the orography.

\subsection{Cloud microphysics and droplets in the turbulent velocity field}

Here we present two examples of cloud microphysical properties representative of the variety of conditions typical at the UFS. On 11 August 2009 the UFS was exposed to relatively intermittent, thin clouds for several hours, from which a 40 min time period was selected. The data were recorded at 17:00 UTC. On 26 October 2009 the UFS was embedded in thick clouds for an extended period of time, and a $100 \mathrm{~min}$ time series of homogeneous coverage was selected which was recorded at around 08:00 UTC. On both days the flow 
was characterized by westerly directions. The temporal evolution of the cloud droplet size distribution is shown in Fig. 8. The 11 August period is dominated by extremely small cloud droplets, with intermittent bursts of droplets with diameters of approximately 8 to $12 \mu \mathrm{m}$, representative of microphysical conditions in small cumulus or thin stratocumulus clouds, either just in the process of formation or during dissipation (Lehmann et al., 2009; Ditas et al., 2012). The 26 October period shows a much more symmetric size distribution with a mean-diameter mode varying between 12 and $14 \mu \mathrm{m}$.

Time-averaged probability density functions for the two periods are shown in Fig. 9. Estimation of the size distribution from the PICT requires normalization by the dropletsize-dependent detection cross section, which is obtained directly from the distribution of beam-transit times (Chuang et al., 2008). Specifically, the droplet number $N_{k}$ in the $k$ th size bin of width $\Delta$ is calculated following $N_{k}=(1 / \Delta)$. $\left(\widetilde{N}_{k} / d_{\text {beam }, k}\right) / \sum_{k}\left(\widetilde{N}_{k} / d_{\text {beam }, k}\right)$, where $d_{\text {beam, },}$ is the effective beam diameter for the $k$ th size bin and $\widetilde{N}_{k}$ is the sampled number of droplets in the $k$ th size bin. Immediately striking are the pronounced exponential large-droplet tails. It is apparent especially in the 26 October example that some mechanism for large-droplet production was likely present, given the small but significant number of droplets with diameters above $25 \mu \mathrm{m}$. Given that peak liquid water contents for that date are only approaching $0.5 \mathrm{~g} \mathrm{~m}^{-3}$, it is not obvious that coalescence is the source of large droplets: the large droplets are still too small to experience significant settling from higher levels in the cloud, but there are also indications that mixing and dilution have taken place, suggesting that the liquid water content could have been higher earlier in the evolution of the cloud. We speculate, however, that mechanisms related to turbulent mixing may be important, including the production of large droplets within diluted cloud regions (Cooper et al., 2013) or through vertical cycling on timescales shorter than the phase relaxation time (Korolev et al., 2013). We will consider aspects of these two mechanisms later in this section, specifically, signatures of homogeneous vs. inhomogeneous mixing, and estimation of the phase relaxation time.

The microphysical conditions sampled in these two examples are quite representative of those measured in free clouds (Lehmann et al., 2009; Ditas et al., 2012). We may also consider which part of the dimensionless parameter space they lie in with respect to droplet inertia and sedimentation effects relevant to particle-turbulence interactions (e.g., Siebert et al., 2010a). The importance of droplet inertia in response to a turbulent flow can be quantified by the Stokes number:

$\mathrm{St}=\frac{\tau_{d}}{\tau_{\eta}}=\frac{\rho_{w}}{\rho_{a}} \frac{d^{2}}{18} \sqrt{\frac{\varepsilon}{v^{3}}} \propto d^{2} \sqrt{\varepsilon}$,
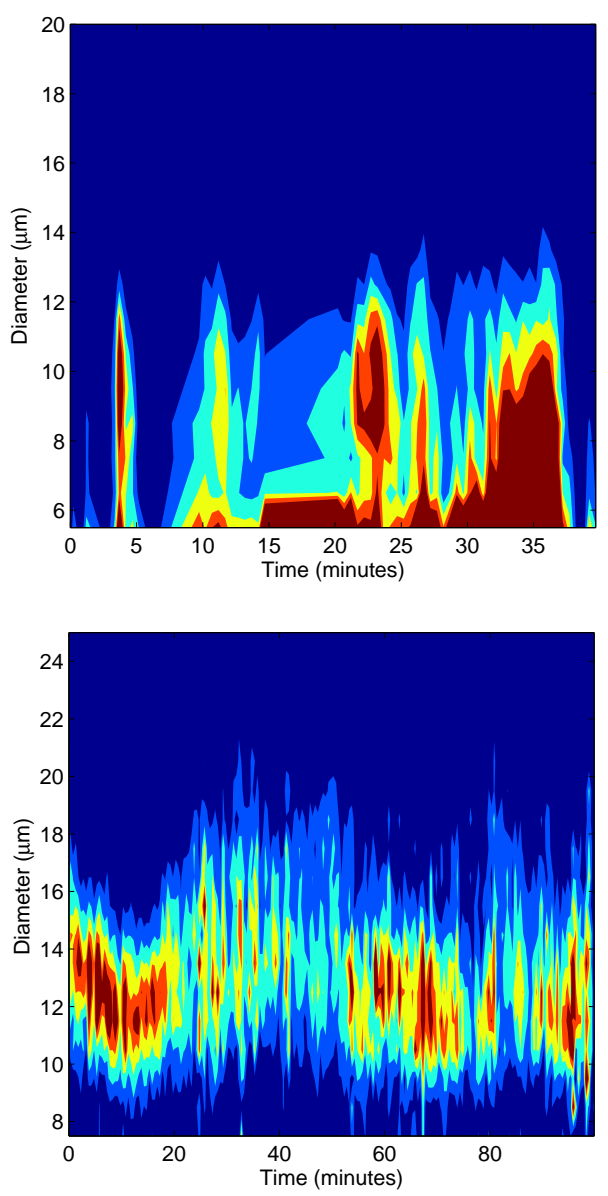

Figure 8. Temporal evolution of the cloud droplet size distribution during a 40 min time period on 11 August 2009 (top) and a $100 \mathrm{~min}$ period on 26 October 2009 (bottom). The color scheme corresponds to droplet number density. The size distributions were measured by the phase Doppler interferometer (PICT).

where $\tau_{\eta}=(\nu / \varepsilon)^{1 / 2}$ is the Kolmogorov timescale and $\tau_{d}=$ $\left(\rho_{w} / \rho_{a}\right)\left(d^{2} / 18 v\right)$ is the inertial droplet response time. The relative importance of gravitational settling can be quantified through the ratio of terminal velocity $v_{t}=g \tau_{d}$ and Kolmogorov velocity $v_{\eta}=(\nu \varepsilon)^{1 / 4}$ :

$\mathrm{Sv}=\frac{\tau_{d} \cdot g}{v_{\eta}}=\frac{\rho_{w}}{\rho_{a}} \frac{d^{2} g}{18 \varepsilon^{1 / 4} v^{5 / 4}} \propto \frac{d^{2}}{\varepsilon^{1 / 4}}$

Figure 10 shows a scatterplot of $(\mathrm{St}, \mathrm{Sv})$ points for both cloud samples, where each point represents an average over $1 \mathrm{~s}$. The mean droplet diameter $\bar{d}$ over the $1 \mathrm{~s}$ period has been calculated by applying the appropriate sample-volume correction $\bar{d}=\sum_{i}\left(d_{i} / d_{\text {beam }, i}\right) / \sum_{i}\left(1 / d_{\text {beam }, i}\right)$. The turbulent energy local dissipation rate is obtained from the sonic data, using similar processing as described by Siebert et al. (2006b).

Although the energy dissipation rates are quite similar for both measurement periods (see middle panel of Fig. 9), the two scatterplots significantly differ due to the contrasting 

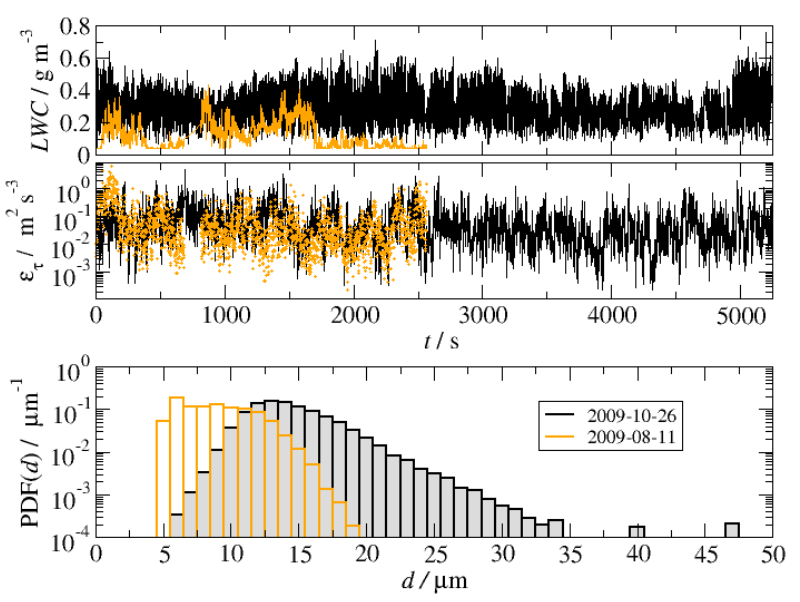

Figure 9. Time series of liquid water content LWC (top panel), local energy dissipation rate $\varepsilon$ (middle panel), and probability density function for the droplet diameter $\operatorname{PDF}(d)$ (lower panel) as observed during the $100 \mathrm{~min}$ long cloud period on 26 October 2009 (black lines) and the $40 \mathrm{~min}$ long record on 11 August 2009 (orange lines).

microphysical conditions: both St and Sv have $d^{2}$ dependence, resulting in relatively high sensitivity to the distinct mean droplet diameters. However, both data sets show similar values as found by Siebert et al. (2010a) for free continental clouds (not shown here). For contrast, two examples of a maritime cloud situation with larger droplets, measured during the CARRIBA campaign, are also shown in Fig. 10. These cloud data were sampled in shallow trade wind cumuli under clean conditions, and the large-droplet diameters yield significantly higher St and Sv. This allows us to conclude that, from the perspective of fine-scale droplet-turbulence interactions, cloud droplets in a turbulent flow at the UFS are representative of free-atmospheric clouds in a continental environment.

We now consider two further ways in which the turbulence can interact with cloud microphysics. First, turbulence leads to mixing between the cloud and clear-air environment, with corresponding reduction in liquid water content. That reduction, however, can appear in either the droplet number density $n$ or the mean droplet diameter $d$, and the details of how it occurs give some hints as to the type of mixing that is occurring (e.g., Jensen et al., 1985). For homogeneous mixing, both $n$ and $d$ are reduced monotonically, as for a population of droplets exposed to identical (well-mixed) thermodynamic conditions. For inhomogeneous mixing, some subset of the droplets is exposed to subsaturated conditions and evaporates completely, while the majority of the droplets experience no evaporation. The result is a reduction in $n$ but little or no reduction in $d$. The homogeneous limit can be calculated if the environmental humidity is known, but in this case it is not, so we plot $d$ vs. $n$ and consider qualitatively whether the measured number densities and droplet sizes (for equaltime bins) tend to prefer one monotonic decrease of $n$ and

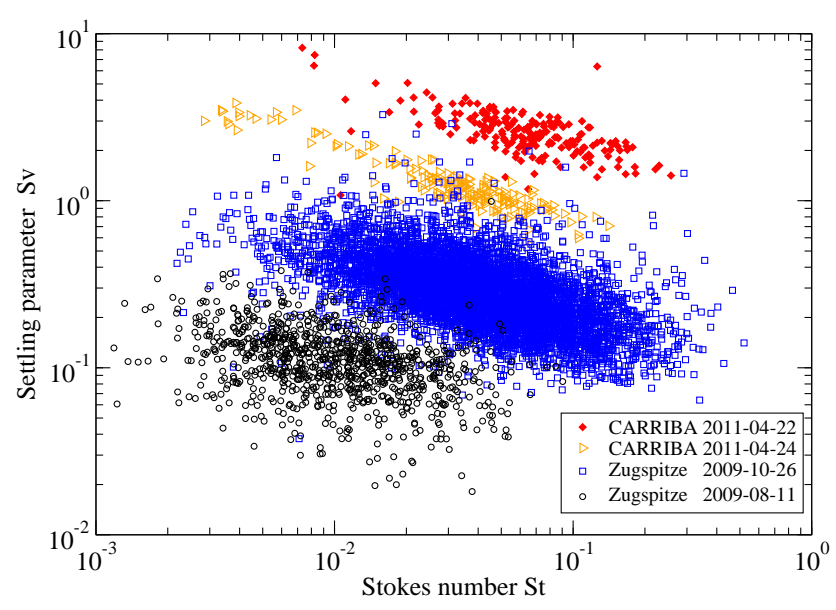

Figure 10. Dimensionless Stokes and settling parameter space. Each $(\mathrm{St}, \mathrm{Sv})$ point is based on a $1 \mathrm{~s}$ average of cloud data. The CARRIBA data represent typical conditions for clean (red) and slightly more polluted (yellow) cases and provide a reference for typical trade wind cumuli (see Siebert et al., 2013, for more details). Data from small cumulus and stratocumulus clouds under continental conditions are not shown but are nearly coincident with the Zugspitze values (Siebert et al., 2010a).

$d$, or decrease of $n$ without $d$. We show data from the long time record taken on 26 October in Fig. 11. Both $n$ and $d$ are obtained from the PICT measurements of the droplet size distribution, and each point in the figure corresponds to a $2 \mathrm{~s}$ average (approximately $8 \mathrm{~m}$ length). The data show wide variation in $n$ with an almost uniform value of $d$, clearly suggesting prevalence of inhomogeneous mixing.

The second question is concerning the range of timescales for turbulent eddies within the inertial subrange, relative to the cloud supersaturation, or phase relaxation time. Specifically, it has been suggested by Mazin (1999), with empirical support from Davis et al. (1999) and Gerber et al. (2001), that the smallest high-frequency eddies should experience larger variance in microphysical properties. This follows the qualitative argument that for eddy timescales of the same order as or less than the phase relaxation time, the supersaturation is not able to reach its quasi-steady value for a given vertical velocity. Therefore, droplets will grow in an environment different than that typically assumed in steady-state models. It has been suggested that this can lead to broadening of the droplet size distribution (Korolev et al., 2013).

We have selected two subsets of the 11 August and 26 October data sets (cf. Fig. 9), for calculation of power spectral density of liquid water content. Special care was taken to ensure a steady mean wind direction from west to minimize possible bias of the liquid water statistics from air not entering the PVM directly through its opening. The length of the time series was $130 \mathrm{~s}$ for 11 August and $65 \mathrm{~s}$ for 26 October, and the LWC data were processed at the full sampling frequency of $1 \mathrm{kHz}$. The power spectra are shown in Fig. 12, and 


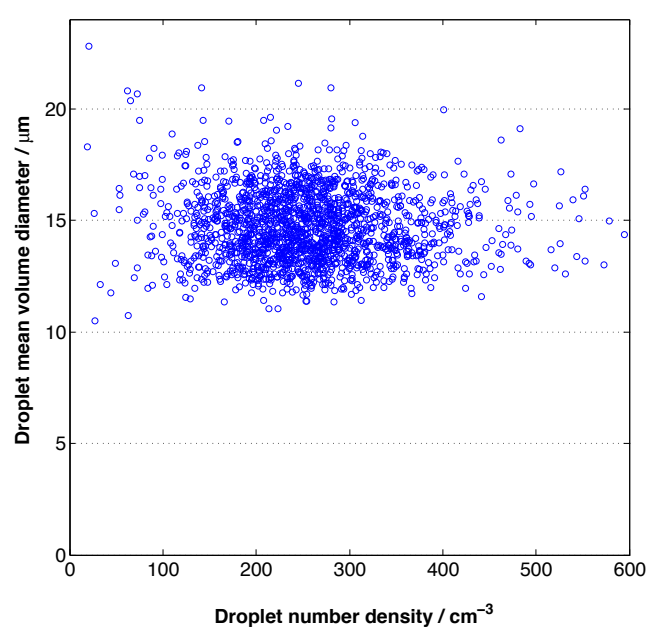

Figure 11. Mean-volume droplet diameter vs. droplet number density for $2 \mathrm{~s}$ intervals in the 26 October 2009 data set. The nearly horizontal distribution of points is indicative of inhomogeneous mixing.

the flattening of the spectra at high frequencies is clearly evident. Following the argument of Mazin (1999), we estimate the transition frequency for this flattening and use the mean flow speed and Taylor's hypothesis to convert the frequency to an eddy length scale. This length scale can be compared to an eddy length scale estimated from the phase relaxation time, which is independently calculated from direct measurement of the droplet size distribution with the phase Doppler interferometer. Using the measured turbulent kinetic energy dissipation rate and Kolmogorov scaling for the inertial subrange, this timescale is converted to an eddy length scale for phase relaxation.

The liquid water content power spectra in Fig. 12 show approximate transitions at frequencies of 2 to $3 \mathrm{~Hz}$ for both dates. The mean flow speeds during the subrecords are $4.2 \mathrm{~m} \mathrm{~s}^{-1}$ for 11 August and $3.6 \mathrm{~m} \mathrm{~s}^{-1}$ for 26 October, leading to length scales of 1.8 and $1.4 \mathrm{~m}$, respectively. The phase relaxation time can be estimated as $\tau_{\text {phase }}=\left(2 \pi D^{\prime} n \bar{d}\right)^{-1}$, where $n$ is the droplet number density, $\bar{d}$ is the mean droplet diameter, and $D^{\prime}$ is a modified diffusion coefficient for water vapor in air (accounting for thermal transport limitations). For the 11 August subrecord corresponding to Fig. 12, the PICT data give $n=532 \mathrm{~cm}^{-3}$ and $\bar{d}=9.9 \mu \mathrm{m}$, resulting in $\tau_{\text {phase }} \approx 2.9 \mathrm{~s}$. For the 26 October subrecord the values are $n=275 \mathrm{~cm}^{-3}$ and $\bar{d}=12.9 \mu \mathrm{m}$, resulting in $\tau_{\text {phase }} \approx 4.7 \mathrm{~s}$. The dissipation rates for the 11 August and 26 October are both $\varepsilon=0.1 \mathrm{~m}^{2} \mathrm{~s}^{-3}$, and using $l_{\text {phase }} \approx\left(\varepsilon \tau_{\text {phase }}^{3}\right)^{1 / 2}$ we obtain $l_{\text {phase }}=1.6 \mathrm{~m}$ and $l_{\text {phase }}=3.2 \mathrm{~m}$, respectively. These are reasonably close to the length scales of 1.8 and $1.4 \mathrm{~m}$ from the observed liquid water content power spectra. This is the first effort to make independent measurements of the transition frequency and of the microphysical parameters necessary to calculate the phase relaxation time, and the results are supportive of the arguments of Mazin (1999).

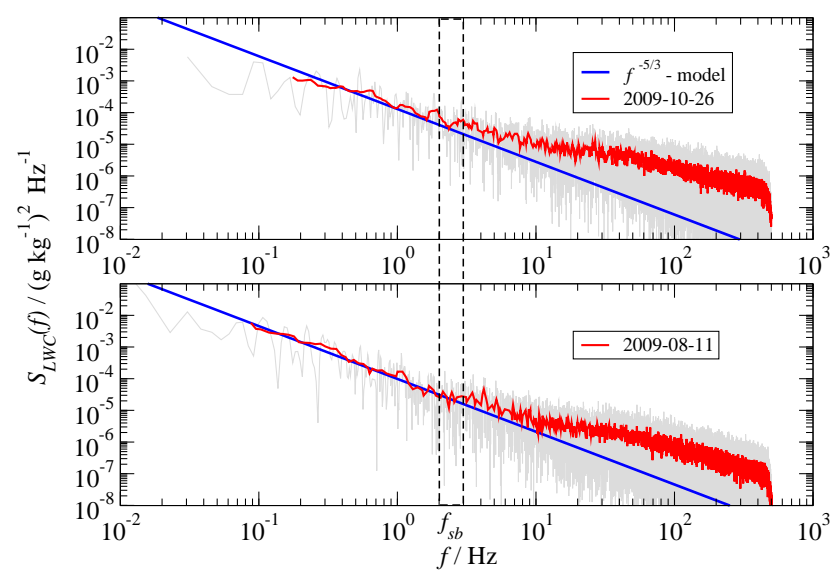

Figure 12. Power spectral densities of LWC for the $100 \mathrm{~min}$ long cloud period on 26 October 2009 (upper panel) and the $40 \mathrm{~min}$ long record on 11 August 2009 (lower panel). The light grey lines indicate the raw spectra, whereas the red lines indicate a averaged spectra. The solid blue line represents a $-5 / 3$ slope for inertial subrange scaling. The vertical lines denote the scale that corresponds to the phase relaxation time estimated from the phase Doppler measurements.

A second scale break is obvious around 30 to $40 \mathrm{~Hz}(\sim$ $10 \mathrm{~cm}$ ), which we cannot explain. However, a similar behavior of LWC spectra has been observed in free clouds by Gerber et al. (2001). In general, the PVM-100A can resolve structures down to the centimeter scale (cf. Fig. 3 in Siebert et al., 2003), so we consider this second scale break as real and not as an instrument limitation.

\section{Conclusions}

High-spatial-resolution measurements of liquid water content, droplet diameter, and turbulent velocity fluctuations were made at the environmental research station UFS in summer and fall 2009. Three-dimensional ultrasonic and onecomponent hot-wire velocity measurements are analyzed to provide a detailed view of small-scale turbulence. Results are reasonably consistent with the assumption of statistically stationary and isotropic turbulence on the spatial scales of relevance to cloud microphysical processes. Time series of droplet number density, liquid water content, and local velocity gradients and energy dissipation rate, as well as averaged cloud droplet size distributions, are very comparable with measurements of boundary-layer-topped cloud properties from prior ACTOS field projects under continental conditions. Comparisons of relevant dimensionless parameters for droplet inertial effects, the Stokes number, and the settling parameter show similar ranges as in free clouds, suggesting that the mountaintop station is a reasonable location for making measurements of cloud-turbulence interactions.

This work was motivated primarily by the desire to make cloud-turbulence measurements that would be difficult to ob- 
tain from an airborne system. Specifically, techniques for Lagrangian tracking of particles in turbulent flows have provided new perspectives on the behavior of inertial particles in turbulence (Ayyalasomayajula et al., 2006; Gibert et al., 2012; Bewley et al., 2013). Of course, there are deviations from idealized flow conditions achievable in a laboratory setting: for example, boundary conditions vary in time so that quasi-stationary conditions must be sought. The data show, however, that stationary subrecords exhibit the fine-scale properties that would be encountered in idealized laboratory flows (e.g., Siebert et al., 2010b). And there are deviations from conditions predominating in free clouds, such as the possible presence of periodicity arising from boundary-layer effects (cf. Fig. 6). Nevertheless, the measurements reported here, together with the work of Risius et al. (2015), generally support the argument that the UFS is a suitable location for detailed Lagrangian measurements of cloud droplets in turbulence, both of which have properties representative of free clouds.

Acknowledgements. We thank Thomas Conrath for help with logistical aspects of the experiment and to Matthew Beals and Amanda Shaw for help with data processing. We are grateful to Markus Neumann and the staff at the UFS for their technical help at the UFS and the Bavarian Environmental Agency (Umweltministerium) for the financial support of the station. Financial support from the Max Planck Society, Deutsche Forschungsgemeinschaft (DFG) through the SPP 1276 Metström, the EU COST Action MP0806 "Particles in Turbulence", and through the US National Science Foundation (NSF grant AGS-1026123) are gratefully acknowledged.

Edited by: M. Wendisch

\section{References}

Ayyalasomayajula, S., Gylfason, A., Collins, L. R., Bodenschatz, E., and Warhaft, Z.: Lagrangian measurements of inertial particle accelerations in grid generated wind tunnel turbulence, Phys. Rev. Lett, 97, 144507, doi:10.1103/PhysRevLett.97.144507, 2006.

Bewley, G. P., Saw, E.-W., and Bodenschatz, E.: Observation of the sling effect, New J. Phys., 15, 083051, doi:10.1088/13672630/15/8/083051, 2013.

Chuang, P. Y., Saw, E. W., Small, J. D., Shaw, R. A., Sipperley, C. M., Payne, G. A., and Bachalo, W.: Airborne Phase Doppler Interferometry for Cloud Microphysical Measurements, Aerosol Sci. Technol., 42, 685-703, 2008.

Comte-Bellot, G.: Hot-wire anemometry, Annu. Rev. Fluid Mech., 8, 209-231, 1976.

Cooper, W. A., Lasher-Trapp, S. G., and Blyth, A. M.: The Influence of Entrainment and Mixing on the Initial Formation of Rain in a Warm Cumulus Cloud, J. Atmos. Sci., 70, 1727-1743, 2013.

Davidson, P. A.: Turbulence, Oxford University Press, Oxford, 657 pp., 2004.
Davis, A. B., Marshak, A., Gerber, H., and Wiscombe, W. J.: Horizontal structure of marine boundary layer clouds from centimeter to kilometer scales., J. Geophys. Res., 104, 6123-6144, 1999.

Ditas, F., Shaw, R. A., Siebert, H., Simmel, M., Wehner, B., and Wiedensohler, A.: Aerosols-cloud microphysicsthermodynamics-turbulence: evaluating supersaturation in a marine stratocumulus cloud, Atmos. Chem. Phys., 12, 2459-2468, doi:10.5194/acp-12-2459-2012, 2012.

Gerber, H.: Direct measurement of suspended particulate volume concentration and far-infrared extinction coefficient with a laserdiffraction instrument., Appl. Opt., 30, 4824-4831, 1991.

Gerber, H., Jensen, J. B., Davis, A. B., Marshak, A., and Wiscombe, W. J.: Spectral density of cloud liquid water content at high frequencies., J. Atmos. Sci., 58, 497-503, 2001.

Gibert, M., Xu, H., and Bodenschatz, E.: Where do small, weakly inertial particles go in a turbulent flow?, J. Fluid Mech., 698, 160-167, 2012.

Gylfason, A., Ayyalasomayajula, S., and Warhaft, Z.: Intermittency, pressure and acceleration statistics from hot-wire measurements in wind-tunnel turbulence, J. Fluid Mech., 501, 213-229, 2004.

Haman, K. E., Makulski, A., Malinowski, S. P., and Busen, R.: A new ultrafast thermometer for airborne measurements in clouds, J. Atmos. Oceanic Technol., 14, 217-227, 1997.

Jensen, J. B., Austin, P. H., Baker, M. B., and Blyth, A. M.: Turbulent mixing, spectral evolution and dynamics in a warm cumulus cloud, J. Atmos. Sci., 42, 173-192, 1985.

Kolmogorov, A. N.: A refinement of previous hyotheses concerning the local structure of turbulence in a viscous incompressible fluid at high Reynolds number, J. Fluid Mech., 13, 82-85, 1962.

Korolev, A., Pinsky, M., and Khain, A.: A New Mechanism of Droplet Size Distribution Broadening during Diffusional Growth, J. Atmos. Sci., 70, 2051-2071, 2013.

Lehmann, K., Siebert, H., and Shaw, R. A.: Homogeneous and inhomogeneous mixing in cumulus clouds: Dependence on local turbulence structure, J. Atmos. Sci., 66, 3641-3659, 2009.

Mazin, I.: The effect of condensation and evaporation on turbulence in clouds, Atmos. Res., 51, 171-174, 1999.

Monin, A. S. and Yaglom, A. M.: Statistical Fluid Mechanics, Volume 2, Dover Publications, Inc Mineola, New York, 874 pp., 2007.

Risius, S., Xu, H., Di Lorenzo, F., Xi, H., Siebert, H., Shaw, R. A., and Bodenschatz, E.: Schneefernerhaus as a mountain research station for clouds and turbulence, 8, 3209-3218, doi:10.5194/amt-8-3209-2015, 2015.

Siebert, H. and Muschinski, A.: Relevance of a Tuning-Fork Effect for Temperature Measurements with the Gill Solent HS Ultrasonic Anemometer-Thermometer, J. Atmos. Oceanic Technol., 18, 1367-1376, 2001.

Siebert, H., Wendisch, M., Conrath, T., Teichmann, U., and Heintzenberg, J.: A new tethered balloon-borne payload for finescale observations in the cloudy boundary layer, Bound.-Lay. Meteorol., 106, 461-482, 2003.

Siebert, H., Franke, H., Lehmann, K., Maser, R., Saw, E. W., Schell, D., Shaw, R. A., and Wendisch, M.: Probing Fine-Scale Dynamics and Microphysics of Clouds with Helicopter-Borne Measurements, B. Am. Meteorol. Soc., 87, 1727-1738, 2006a.

Siebert, H., Lehmann, K., and Wendisch, M.: Observations of small scale turbulence and energy dissipation rates in the cloudy boundary layer., J. Atmos. Sci., 63, 1451-1466, 2006 b. 
Siebert, H., Lehmann, K., and Shaw, R.: On the use of a hot-wire anemometer for turbulence measurements in clouds, J. Atmos. Oceanic Technol., 24, 980-993, 2007.

Siebert, H., Gerashchenko, S., Lehmann, K., Gylfason, A., Collins, L. R., Shaw, R. A., and Warhaft, Z.: Towards understanding the role of turbulence on droplets in clouds: In situ and laboratory measurements, and numerical modeling, Atmos. Res., 97, 426437, 2010a.

Siebert, H., Shaw, R. A., and Warhaft, Z.: Statistics of small-scale velocity fluctuations and internal intermittency in marine stratocumulus clouds, J. Atmos. Sci., 67, 262-273, 2010 b.
Siebert, H., Beals, M., Bethke, J., Bierwirth, E., Conrath, T., Dieckmann, K., Ditas, F., Ehrlich, A., Farrell, D., Hartmann, S., Izaguirre, M. A., Katzwinkel, J., Nuijens, L., Roberts, G., Schäfer, M., Shaw, R. A., Schmeissner, T., Serikov, I., Stevens, B., Stratmann, F., Wehner, B., Wendisch, M., Werner, F., and Wex, H.: The fine-scale structure of the trade wind cumuli over Barbados - an introduction to the CARRIBA project, Atmos. Chem. Phys., 13, 10061-10077, doi:10.5194/acp-13-10061-2013, 2013.

Whitemann, C. D.: Mountain Meteorology, Oxford University Press, Oxford, 355 pp., 2000.

Willis, G. E. and Deardorff, J. W.: On the use of Taylor's translation hypothesis for diffusion in the mixed layer, Q. J. Roy. Meteor. Soc., 102, 817-822, 1976. 